\title{
El argumento de responsabilidad social de la industria tabacalera en Brasil
}

\author{
Tânia Cavalcante, MD, MPH,(1) Aline de Mesquita Carvalho, MD,(1) Erica Cavalcanti Rangel, MD, MSP.(I)
}

\author{
Cavalcante T, De Mesquita Carvalho A, \\ Cavalcanti Rangel E. \\ El argumento de la responsabilidad social \\ de la industria tabacalera en Brasil. \\ Salud Publica Mex 2006;48 supl I:SI73-SI82.
}

\section{Resumen}

El tema de la responsabilidad social corporativa ha sido asunto de gran importancia y preocupación en el mundo actual y ha recibido diferentes denominaciones, tales como responsabilidad social, ciudadanía corporativa, desarrollo sustentable o ética corporativa. Hoy más que nunca se hace necesario que órganos gubernamentales, así como las diversas representaciones de la sociedad civil organizada, actúen alineados con el concepto de desarrollo sustentable. Esto implica el entendimiento de que, junto con la preservación del medio ambiente, la salud y la educación representan la base de la productividad económica. Significa entender, asimismo, que una población saludable es esencial para la reducción de la pobreza, el crecimiento económico y el desarrollo sustentable. Las diferentes experiencias positivas de Brasil en el enfrentamiento de las estrategias de la industria tabacalera para minar los esfuerzos nacionales del control del tabaquismo tiene como base una amplia articulación en red. Esta red ha ejercido un papel fundamental de control social en el monitoreo y fiscalización de las políticas públicas para el control del tabaco, así como de las estrategias de la industria tabacalera.

Palabras clave: responsabilidad social; industria tabacalera; Brasil
Cavalcante T, De Mesquita Carvalho A,

Cavalcanti Rangel E.

Social responsibility argument

for the tobacco industry in Brazil.

Salud Publica Mex 2006;48 suppl I:SI73-SI 82.

\section{Abstract}

The issue of "corporate social responsibility" has been one of great importance and concern in the world and has received different names, such as social responsibility, corporate citizenship, sustainable development and corporate ethics. Today, more than ever, it has been necessary for governments, as well as the diverse representatives of "organized civil society," to act in accordance with the concept of sustainable development. This implies an understanding that preservation of the environment, health and education is related to economic productivity; it means an understanding that healthy populations are essential to the reduction of poverty, as well as economic growth and sustainable development. The various positive experiences in Brazil in confronting the tobacco industry's strategies to undermine national tobacco control efforts are due to the existence of a wide network that has played a fundamental role in social control with respect to both the monitoring of public policies that control tobacco and tobacco industry strategies.

Keywords: social responsibility; tobacco industry; Brazil
A ctualmente el tabaquismo es considerado un problema grave de salud pública por la dependencia química que provoca y por actuar como factor de riesgo en casi 50 enfermedades diversas, entre las que destacan las cardiovasculares, las respiratorias crónicas obstructivas y el cáncer. ${ }^{1-4}$
Sin embargo, el conocimiento acumulado no ha sido suficiente para minimizar el consumo de productos de tabaco en el mundo, especialmente del cigarro. Por el contrario, éste se ha acrecentado de forma alarmante, sobre todo en países en desarrollo, como resultado de estrategias globales sofisticadas y bien articuladas, des-

(I) División del Control del Tabaquismo y otros factores de riesgo, Instituto Nacional del Cáncer, Secretaría de Salud. Brasil.

Fecha de aprobado: 19 de abril de 2006

Solicitud de sobretiros: Instituto Nacional de Câncer (INCA). Praça Cruz Vermelha 23, Centro 20230-I50. Río de Janeiro, Brasil.

Correo electrónico:tania@inca.gov.br 
plegadas por grandes compañías trasnacionales que promueven y venden estos productos. Favorecidas por la globalización de la economía, estas compañías han provocado un rápido desplazamiento de la epidemia del tabaquismo de los países ricos a los países pobres. Actualmente, cerca de $20 \%$ de la población mundial es fumadora, y $80 \%$ de los fumadores se concentran en países en vías de desarrollo. ${ }^{5}$

Con ello, una carga económica y social cada vez más pesada se está imponiendo en innumerables países de media y baja liquidez, muchos de los cuales aún luchan para controlar enfermedades transmisibles, reducir la desnutrición y las tasas de mortalidad infantil. Este escenario ha llevado a diversas instituciones derivadas de la Organización de las Naciones Unidas (ONU) a la conclusión de que el tabaquismo es también un factor que contribuye al aumento de la pobreza e impide el desarrollo sustentable. ${ }^{6}$ De hecho, con el objetivo de crear conciencia sobre esta dimensión del problema de tabaquismo, la Organización Mundial de la Salud (OMS) abordó en el 2004 el tema "Tabaco y Pobreza: un ciclo vicioso", para la campaña de "Un Día Mundial Sin Tabaco". ${ }^{\prime}$

Todo esto también indica que el control global del consumo de tabaco depende del control de sus causas, incluso de las industrias trasnacionales. En este sentido, la amenaza que la dinámica del libre comercio e inversión sitúa sobre las iniciativas nacionales de control está en la mira y en la preocupación de las futuras Asambleas Mundiales de la Salud (AMS). Así, en 1978, la AMS expresaba, en una de sus resoluciones, su preocupación por " [...] un alarmante crecimiento en la producción y en el consumo de cigarros durante las dos últimas décadas en los países en vías de desarrollo, en los cuales el consumo no había sido incrementado, y [por] cómo el amplio direccionamiento promocional para la venta de cigarros se estaba desarrollando en los medios de comunicación, y de la asociación de estos productos con eventos culturales y deportivos, generalmente induciendo a los jóvenes a fumar". ${ }^{8}$

El creciente conocimiento de la gravedad de esta práctica llevó al Banco Mundial a prohibir en 1991 la utilización de sus recursos para el financiamiento del producto de tabaco, y a estimular en los países la suma de esfuerzos para controlar su consumo como parte de una estrategia a favor del desarrollo sustentable y de lucha contra la pobreza. ${ }^{9}$ En este sentido, el Banco Mundial recomendó una serie de medidas prácticas para la reducción del tabaquismo como: a) prohibición de propaganda; b) medidas en el incremento de impuestos y precios derivados del tabaco; c) advertencias sanitarias en el embalaje del producto; y d) educación e información en el tratamiento de la dependencia de la nicotina.
Asímismo, también llamó la atención el argumento de que en muchos países se considera que el tabaco contribuye a la recaudación de impuestos y a la generación de empleos, lo que supone uno de los más grandes desafíos para el control del tabaquismo. ${ }^{10}$

Alarmados con este escenario, en mayo de 1999, durante la 52a Asamblea Mundial de la Salud, 191 países propusieron unánimemente la adopción del Primer Tratado Internacional de Salud Pública, bajo el auspicio de la OMS. Este Tratado, en el Convenio Marco de la OMS para el Control del Tabaco (CMCT), propone la adopción de un conjunto de medidas intersectoriales para detener la expansión global de su consumo y sus nocivas consecuencias. ${ }^{11}$ La evidencia que sustentó dicha decisión fue el reconocimiento de que el tabaco es un producto que mata prematuramente a la mitad de sus consumidores y que no permite un nivel seguro de consumo, sumado al hecho de que la apertura del comercio ha contribuido a expandir globalmente el problema, principalmente en los países en vías de desarrollo. El tratado entró en vigor en febrero del 2005, después de que 40 países ratificaran su adhesión al mismo, e hizo historia como el Tratado de la ONU que más rapidamente captó afiliados y entró en vigor. De hecho, el 8 de abril del 2006 ya contaba con 124 ratificaciones.

Dado lo complicado del tema, en 1999, cuando se iniciaron las discusiones para la negociación en la Convención Marco, se creó una fuerza laboral para el control del tabaquismo, denominada United Nations Ad Hoc Interagency Task Force on Tobacco Control (Fuerza laboral de las Naciones Unidas) -UNTF, por sus siglas en inglés-, a través de la resolución 1999/56 del Consejo Económico y Social de las Naciones Unidas (ECOSOC) de fecha 30 de julio. Como miembros de esa fuerza laboral estan la FAO, la OIT, la UNICEF y el Banco Mundial, entre otros, fungiendo la OMS como Secretaría Ejecutiva. ${ }^{12}$ En diciembre del 2005 se llevó a cabo la 6 a sesión de la "Fuerza Laboral de las Naciones Unidas", teniendo como principales puntos de discusión el nexo entre control del tabaquismo y desarrollo económico, así como las gestiones para la implementación del Convenio Marco en diversos países. En este contexto, una importante recomendación de la UNTF fue que hubiese una gran vigilancia a la interacción de las Naciones Unidas con la industria tabacalera. ${ }^{13}$

En la $11^{\text {a }}$ Conferencia Cuadrienal de las Naciones Unidas sobre Comercio y Desarrollo (UNCTAD XI), ${ }_{14}^{14}$ levada a cabo en Brasil en junio del 2004, se abordó el tema del control del tabaco como un importante punto para los planes de desarrollo de los países, y se generó un documento que llama la atención sobre el impacto negativo que el consumo del producto del tabaco trae para la economía. El documento invita a los estados miembros de 
las Naciones Unidas a fortalecer las medidas para el control del tabaco en sus programas nacionales, y como parte de la implementación de sus planes de desarrollo.

Así, en el 2004, el ECOSOC adoptó una nueva resolución ${ }^{15}$ en donde reconoce el impacto negativo del tabaquismo en la salud, la economía, el desarrollo y el medio ambiente, y resalta la necesidad de que las acciones para su control estén integradas en los planes de combate a la pobreza. También hace énfasis en la necesidad de movilizar los recursos para el fortalecimiento de las acciones de control del tabaquismo, principalmente en los países en vías de desarrollo, convocando a todos los países a adherirse a la Convención Marco de la OMS para el Control del Tabaco. Asimismo, abre la invitación a todas las agencias, fondos y programas de las Naciones Unidas y otros organismos internacionales, para que apoyen dichas iniciativas en el ámbito nacional e internacional.

\section{Estrategias de la industria tabacalera para lidiar con un escenario cada vez más hostil}

Desde que cientos de documentos internos de la industria tabacalera fueron publicados a partir de acuerdos judiciales en el sistema legal de Estados Unidos de América y del Reino Unido en 1999, la comunidad internacional de la salud pública tomó conocimiento de las estrategias y prácticas desleales adoptadas por grandes compañías trasnacionales de tabaco para mantener una expansión del consumo de sus productos y, con él, de todos los males que causa (Legacy Tobacco Document Library). ${ }^{16}$ Entre las diversas estrategias descritas en estos documentos destacan las que tienen como objetivo impedir o revertir acciones de control del tabaquismo en países del mundo entero, incluidos los de Latinoamérica.

Algunos párrafos de los documentos analizados muestran el pensamiento estratégico de grandes compañías tabacaleras en relación con los mercados de los países en vías de desarrollo.

- El consumo de tabaco en las naciones desarrolladas seguirá una tendencia descendente hacia el final del siglo, mientras que en los países en desarrollo el consumo podría aumentar aproximadamente en un tres por ciento anual. ¡Un cuadro verdaderamente prometedor! No habrá una sociedad sin fumadores, sino un crecimiento sostenido para la industria tabacalera. (Tobacco Reporter, 1989 apud Campaign for Tobacco Free Kids)

- No deberíamos sentirnos deprimidos simplemente porque el mercado total del mundo libre parece disminuir. Dentro del mercado total, hay áreas de sólido crecimiento, particularmente en Asia y África; se abren nuevos mercados a nuestras exportaciones, tales como en los países del área Indo-China y del Comecón; y hay grandes oportunidades de incrementar nuestra participación en el mercado en áreas como Europa.... Esta industria es sistemáticamente rentable. Y hay oportunidades de aumentar aún más esa rentabilidad. (BAT, 1990 apud Campaign for Tobacco Free Kids)

- La expectativa de vida media aquí es de unos 40 años, la mortalidad infantil es alta: los problemas de salud que algunos dicen son causados por los cigarrillos realmente no se percibirán como un problema aquí. (Según el representante de Rothmans en Burkina Faso 1988 apud Campaign for Tobacco Free Kids)

- Este es un mercado con un enorme potencial. El índice de crecimiento demográfico es 2,2 por ciento por año y 40 por ciento de la población es menor de 18 años. (Análisis de la Philip Morris sobre el potencial de mercado en Turquía, 1997 apud Campaign for Tobacco Free Kids)

- Debemos tratar de detener los programas dirigidos a un compromiso del Tercer Mundo en contra del tabaco. Debemos tratar de lograr que todos o al menos una gran parte de los países del Tercer Mundo se comprometan con nuestra causa. Debemos tratar de influenciar la política oficial de la FAO y de UNCTAD para que adopten una postura a favor del tabaco. Debemos tratar de mitigar el impacto de la OMS, forzándola a adoptar una postura más objetiva y neutral. (BAT, 1979 apud Campaign for Tobacco Free Kids)

Un análisis de documentos como estos hacen que dichas estrategias sean vistas como un problema de salud pública. En mayo del 2001, en la 54 ${ }^{a}$ Asamblea Mundial de la Salud (AMS) se expresó formalmente esta preocupación en la resolución 18:8 "Transparencia en el proceso del control del tabaco". En este documento, la AMS incide en la importancia de que la OMS y sus estados miembros se mantengan alerta a cualquier esfuerzo emprendido por la compañías tabacaleras para el mantenimiento de esas prácticas y que, además, redoblen su fuerza para proteger la integridad del desarrollo de las políticas de salud de la OMS y los gobiernos nacionales. También recomienda extremar la atención ante cualquier asociación entre la industria del tabaco y los miembros delegacionales de la OMS. Solicita, además, que la OMS continúe informando a sus estados miembros sobre las actividades de la industria tabacalera, sobre todo de aquellas que tengan impacto negativo en los esfuerzos por controlar el consumo del tabaco. Desde entonces, la OMS tiene monitoreada las estrategias de la industria del 
tabaco mundial y ha divulgado regularmente informes sobre las mismas. ${ }^{17}$

En este sentido, los países miembros negociaron el Convenio Marco de la OMS para el Control del Tabaco, que incluye como artículo de obligación general el compromiso de proteger sus políticas públicas relacionadas con el control del tabaco de los intereses comerciales y de otros intereses defendidos por la industria tabacalera:

- A la hora de establecer y aplicar sus políticas de salud pública relativas al control del tabaco, las Partes [sic] actuarán de una manera que proteja dichas políticas contra los intereses comerciales y otros intereses creados de la industria tabacalera, de conformidad con la legislación nacional. (Convenio Marco para Control del Tabaco. Artículo 5.3)

\section{La industria del tabaco y sus estrategias para influir en las políticas nacionales sobre el control del tabaquismo}

Después de la difusión de estos documentos, grandes industrias tabacaleras adoptaron estrategias para lidiar con un ambiente cada vez más hostil en sus negocios a través de una amplia inversión en mercadotecnia. Los esfuerzos de reivindicación de su imagen incluyen un deseo de diálogo con los diversos sectores gubernamentales para la apertura de reglamentaciones "racionales" a través de acuerdos voluntarios y mercadotecnia social, entre los que destaca su apoyo a proyectos de defensa del medio ambiente y causas sociales, como el combate a la pobreza, explotación infantil y analfabetismo. ${ }^{18}$ Estos esfuerzos buscan revestir las compañías tabacaleras con una imagen positiva de corporaciones socialmente responsables, además de tener al mismo tiempo gran influencia en la opinión pública e incluso en los resultados electorales. Asimismo, a través de estas estrategias de reconstrucción de imagen las empresas actúan sutilmente para conquistar a los formadores de opinión, seducir o presionar a políticos, parlamentarios y gestores, instituciones públicas y organizaciones no gubernamentales, así como crear un clima de "buena voluntad política" hacia sus negocios.

- [...] Identificar aquellos miembros del Congreso que debido a su antigüedad en el cargo, nivel jerárquico, etc., sea probable que formen parte de la próxima generación de líderes del Congreso... Deberíamos refinar los programas actuales para ampliar nuestra presencia ante estos miembros, promoviendo reuniones, comidas y eventos para recaudar fondos, además de las necesidades de financiamientos especiales a estos miembros. (To- bacco Institute, 1982 apud Campaign for Tobacco Free Kids, 200199).

- En 1985 estamos trabajando para mejorar aún más nuestro desempeño en la comunicación con los Legisladores. Nuestro enfoque tiene tres aspectos: Primero; vamos a reclutar más y mejores personas y pagarles más si es necesario. Reforzaremos nuestras unidades de Asuntos Corporativos en Bahrain, Estocolmo, Londres, Filipinas y Japón. Posteriormente adoptaremos un método más sistemático de integrar personal más adecuado (Philip Morris, 1985 Campaign for Tobacco Free Kids 2001 ${ }^{19}$ ).

- Dejar a los políticos conocer el lado mezquino de la contra actividad, identificando a un candidato vulnerable, conjuntando esfuerzos de modo que pierda las elecciones, y después discretamente, hacerle saber a otros políticos que nosotros influimos en esa decisión. (Philip Morris, 1987 apud Campaign for Tobacco Free Kids, 2001 ${ }^{19}$ ).

Diversos documentos del British American Tobacco (BAT) indican objetivos y estrategias en su Programa Corporativo de Responsabilidad Social, dejando claro cuáles son las reales motivaciones de las compañías tabacaleras en sus prácticas de mercadotecnia social: ${ }^{20}$

- Aumentar su incorporación e influenciar a reguladores y políticos.

- Promover una reputación positiva a fin de mejorar nuestra capacidad de preparar el ambiente futuro para los negocios.

- Entre los proyectos se encuentra una clínica para el diagnóstico de enfermedades, casa-hogar para personas sin techo, así como el patrocinio de programas de arte y educación. Para la BAT tales programas son conquistas aliadas en el mercado local para abrir las puertas a políticos y reguladores.

- [...] A través del establecimiento pro activo de nuevos patrones de mercadotecnia internacional para el tabaco, las multinacionales podrían tratar de contar con varias propuestas sobre las cuales la OMS podría trabajar para reducir la cantidad de cigarros que se consumen a nivel internacional.

\section{Responsabilidad social y el comportamiento de la industria tabacalera en brasil}

A partir de la década de los ochenta se instalaron en Brasil grandes compañías tabacaleras trasnacionales, principalmente en la región sur, que dominaron toda una cadena de producción, desde la siembra de las hojas del tabaco hasta la producción del cigarro. ${ }^{21}$ 
Como Brasil ofrecía una gran fuente laboral para la producción agrícola del tabaco -gracias a sus tierras fértiles, clima favorable y mano de obra barata- la presencia económica y política de esas grandes trasnacionales se fortaleció a lo largo de los años y supuso un gran obstáculo al control del tabaquismo. No obstante, a pesar de ser Brasil un país en desarrollo, el segundo mayor productor de tabaco y el máximo exportador del mismo, se logró reducir de forma significativa el consumo del tabaco en su población a lo largo de los últimos 15 años. ${ }^{22}$

Actualmente, el mercado interno brasileño de cigarros es una cadena productiva de tabaco dominada por la British American Tobacco a través de su subsidiaria Souza Cruz, la cual está trabajando de forma cada vez más intensa para, por un lado, promoverse como una empresa socialmente responsable y así captar apoyo político para sus negocios ${ }^{23} \mathrm{y}$, por otro, buscar estrategias para frenar los avances nacionales ya conquistados en el ámbito del control del tabaquismo.

\section{La industria del tabaco y la construcción de una imagen socialmente responsable en Brasil}

A través de páginas en internet y de informes sociales con argumentos políticamente correctos e imágenes positivas de promoción y desarrollo sostenible, la representación nacional de la BAT ha intentado construir una imagen de sí misma como corporación socialmente responsable. En esa dirección una de las iniciativas clave fue la creación del Instituto Souza Cruz (cuyo lema es "Educación para el Desarrollo Humano Sostenible"), como una manera de separar sus negocios de sus actividades de mercadotecnia. ${ }^{24}$ Esta institución, que representa los intereses de la BAT, divulga informes de sus actividades de apoyo a numerosos programas sociales tales como: a) el Programa Reforestar, que incentiva la preservación del bosque en las regiones de plantíos de tabaco; b) el Programa Coseche un Maíz y un Frijol antes de Cosechar Tabaco, una orientación a los productores del sur del país para la siembra de granos antes de la siembra de tabaco; c) el Programa Educar, que proporciona una valoración educativa a los agricultores; y d) el Programa Cuidar, con el objetivo de educar en valores e incentivar el respeto a la diversidad, las creencias y las expectativas de las comunidades, incluso a la propia ética. No obstante, el argumento políticamente correcto de la promoción del desarrollo sostenible no resulta válido frente a las actitudes y estrategias adoptadas por las empresas tabacaleras para minimizar la lucha contra las enfermedades y muertes derivadas del consumo de sus productos. A continuación serán presentadas algunas de las tantas situaciones enfrentadas en Brasil que ilustran esta afirmación.
La incoherencia de la imagen socialmente responsable respecto a las actitudes de la empresa

El caso de los programas de prevención del tabaquismo en los jóvenes

A través de la divulgación de esta nueva forma "responsable" de conducir sus actividades, la BAT, tanto en Brasil, como en otras partes del mundo, ha procurado promover el consumo del tabaco como una "elección adulta". En este sentido, ha utilizado campañas de prevención contra el tabaquismo entre los jóvenes, y ha divulgado su posicionamiento con un tono políticamente correcto. Entre los puntos centrales de su actuación está la decisión de enfocar su negocio a un público consumidor adulto y ya fumador y restringir el acceso al tabaco a los menores de 18 años. En opinión de la institución Souza Cruz la decisión de fumar debe ser tomada por adultos informados y maduros para avalar el placer y riesgo asociados al acto de fumar. De hecho, en Brasil, mucho antes de la actual legislación federal que prohíbe la venta de cigarros a menores de 18 años, Souza Cruz colocó en los empaques de sus productos -por iniciativa propia-, que su venta era solamente para mayores de 18 años y que apoyaba programas "para prevenir y eliminar el acto de fumar en infantes y adolescentes". ${ }^{25}$

Sin embargo, esta política potencia la imagen del cigarro como "fruto prohibido" y estimula el deseo adolescente de trasgresión. De hecho, se convierte en la principal motivación del adolescente para fumar, por su deseo de sentirse adulto y como rebeldía y rechazo a los valores del país. Profesionales del área de la publicidad afirman que este tipo de estrategia responde al objetivo de presentar al cigarro como una de las iniciaciones al mundo del adulto, al convertirlo en un símbolo básico del proceso de crecimiento y maduración (Agencia Publicitaria TED BATES, 1975 apud Campaign for Tobacco Free Kids $2001^{19}$ ).

Se suma a esto el hecho de que documentos internos de grandes compañías tabacaleras en litigio revelan que la imagen de la empresa socialmente responsable nada tiene que ver con una intención de mejorar éticamente sus estratégias y actividades, y que en verdad tendría una función de escudo, un elemento preventivo contra medidas gubernamentales que pretenden reducir el consumo y, con ello, perjudicar sus negocios.

- Nuestro objetivo es comunicar que la industria del tabaco no está interesada en que los jóvenes fumen y posicionar a la industria como una corporación altamente responsable en un esfuerzo para repeler nuevos ataques por el movimiento antita- 
baco (Philip Morris-América Latina: Campaña dirigida a los Jóvenes de América Latina, 1993)

- Un programa para disuadir a los adolescentes de fumar (una decisión adulta) podría prevenir o demorar otra reglamentación de la industria tabacalera (Tobacco Institute, 1982, apud Campaign for Tobacco Free Kids $2001^{19}$ ).

- La creciente presión de las fuerzas contra el tabaco en Latinoamérica ha creado la necesidad de explorar varias opciones para contrarrestar la publicidad negativa... Teniendo en cuenta el clima legislativo adverso emergente en la región, tenemos una oportunidad de crear buena voluntad para la industria tabacalera al sacar una campaña pública para desalentar el consumo de cigarrillos juvenil (Philip Morris, 1994 apud Campaign for Tobacco Free Kids 2001 ${ }^{19}$ ).

- Si podemos presentar una legislación proactiva u otra clase de medidas sobre la cuestión del acceso de la juventud a los cigarrillos [...] protegerémos a nuestra industria durante décadas por venir (Philip Morris, 1995, apud Campaign for Tobacco Free Kids, $2001^{19}$ )

El programa juvenil y sus partes individuales apoyan el objetivo del Tobacco Institute para desalentar las injustas restricciones federales, estatales y locales concernientes a la publicidad de cigarrillos a través de las siguientes medidas:

- Reforzar la creencia de que la presión de los padres -no la publicidad- es la causa de que los jóvenes fumen.

- Cautivar el centro político y forzar a quienes están contra el tabaco a un extremo [...]

- Fomentar intensamente la oposición de la industria a que los jóvenes fumen [...]

- Establecer la idea de un programa bien recibido, en crecimiento, alentando una proliferación de proyectos pequeños, locales, y sucesos conjuntos apropiados con otros aliados. Evitar depender de una sola organización. (Tobacco Institute, 1991 apud Campaign for Tobacco Free Kids, 2001 ${ }^{19}$ )

En función de este escenario, la Agencia Nacional de Vigilancia Sanitaria, responsable de la fiscalización y reglamentación de los productos del tabaco en Brasil, publicó en 2003 una resolución (RDC No. 335) que determina la prohibición de las leyendas "Solamente para adultos" y "Producto para mayores de 18 años", hasta entonces colocadas por la industria tabacalera voluntariamente en el empaque de los cigarros. Además, obligó a incluir en los empaques un mensaje dirigido a los vendedores, "Venta prohibida a menores de 18 años", junto con la cita a la ley federal que prohíbe la venta de sustancias psicoactivas para menores de edad y la respectiva precisión de las penalidades para los infractores. ${ }^{26}$ En la misma línea, el Instituto Nacional de Cáncer, organismo de la Secretaría de Salud, y responsable de la articulación del programa brasileño sobre control del tabaquismo, declinó en el 2001 y 2003 la invitación de la institución Souza Cruz para participar en el proceso de su informe social-corporativo y establecer una sociedad para la prevención del consumo entre los jóvenes.

- $\quad[. .$.$] La posición del INCA es de que es imposible$ reconocer y participar del proceso de diálogo propuesto por esta institución (Souza Cruz), así como subsidiar su informe social, cuyas acciones propuestas consideramos paradojas, en virtud de la naturaleza de sus productos y de sus interés empresariales (Río de Janeiro 19 de septiembre del 2003. Dr. José Gomes Temporão, Director General del INCA).

El caso de la acción directa de inconstitucionalidad para la prohibición de la propaganda en Brasil

Según el Banco Mundial las prohibiciones totales de publicidad y promoción de los productos de tabaco son importantes para reducir el consumo en tanto que las prohibiciones parciales tienen un escaso o nulo efecto. ${ }^{27}$ En Brasil, en diciembre del 2000 una prohibición de la propaganda en los grandes medios de comunicación entró en vigor con la aprobación de la ley federal 10.167, la cual restringió la promoción del cigarro y otros productos derivados del tabaco a sus puntos internos de venta, y prohibió a las marcas de cigarro el patrocinio de eventos culturales y deportivos a partir del 1 de enero del 2003. El proyecto de ley 3.156 que dio origen a esta legislación siguió todos los trámites legales. Primero, fue enviado a la Cámara de Diputados y tramitado de mayo a agosto del 2000 con una aprobación de 380 votos a favor y 80 en contra. Después se remitió al Senado Federal, donde pasó tres audiencias públicas conjuntas ante la Comisión de Asuntos Sociales y de Constitución, Cociudadanía y Justicia (CCJ) y recibió 23 enmiendas antes de su aprobación final en diciembre del 2000. Las audiencias públicas realizadas en el ámbito del Congreso Nacional promovieron un amplio debate y garantizaron un proceso transparente y democrático, con la participación de representantes de la Secretaría de Salud, asociaciones y sociedades médicas, Organizaciones No Gubernamentales, la Asociación Brasileña de Emisoras de Radio y Televisión (ABERT), así como representantes de la industria del tabaco, la Asociación de Fumicultores de Brasil (AFUBRA) y el Sindicato de la Industria 
del Humo (Sindifumo), entre otras. Así, en septiembre del 2004, a través de la Confederación Nacional de la Industria (CNI), la Industria del Humo, que denunció la inconstitucionalidad de esa medida, se personó para declarar ante el Supremo Tribunal Federal, con la intención de revertir el importante avance nacional logrado en el control del tabaquismo. ${ }^{28}$ De forma paralela, el Instituto Souza Cruz apoya el financiamiento del proyecto Justicia sin papel, que pretende implantar la automatización del sistema judicial (ingresado en línea el 6 de abril del 2006), ${ }^{29}$ por estar obviamente en conflicto con el comportamiento de conducta ética y de inmunidad en el arbitraje de materia en donde la industria del tabaco ha sido con frecuencia juzgada de acciones de irresponsabilidad civil ${ }^{30, *}$ y también por ser beneficiaria de la ADIN. Esta iniciativa está siendo cuestionada por el Ministerio Público por "ofender la imparcialidad e independencia del Sistema Judiciario y generar dependencia del mismo a costa del sector privado". ${ }^{31}$

El difícil proceso de ratificación del Convenio Marco de la OMS para el control del tabaco en Brasil

Brasil presidió el Organo de Negociación Intergubernamental del CMCT (Intergovernmental Negotiation Body of the Framework Convention on Tobacco Control) durante todo el proceso de su discusión, así como de sus reuniones de taller grupal intergubernamental, que prepara las bases para la primera sesión de la Conferencia de las Partes del CMCT. Contrario a las expectativas de que fuese uno de los primeros países en

\footnotetext{
* Las acciones judiciales contra la Souza Cruz, por supuestos daños atribuibles al consumo de cigarros, se iniciaron en 1995, siguiendo las tendencias de la industria denunciante existente en los Estados Unidos..

Así como acontece en los tribunales americanos y de otros países, al considerar tales solicitudes, el Poder Judicial de Brasil rechaza reiteradamente las acciones, valiéndose básicamente de los siguientes argumentos que defienden a la Souza Cruz

- La fabricación y comercialización de cigarros son actividades lícitas y reglamentadas por el Poder Público.

- Los riesgos atribuibles al consumo de cigarros son del conocimiento del público desde hace décadas, inclusive antes de que se usaran cláusulas de advertencia.

- La propaganda no es engañosa, apenas, como cualquier otra, destaca las características positivas del producto.

- Los fumadores, al optar libremente por el consumo de cigarros, asumen los riesgos consecuentes a su decisión, por lo que no pueden pretender responsabilizar al fabricante por un acto consciente y de libre albedrío.

- Las enfermedades atribuibles al consumo de cigarros son multifactoriales. Existen otros factores que podrían haber generado enfermedades de riesgo tan diverso como la predisposición genética, exposición ambiental, dieta inadecuada, enfermedades preexistentes, estilos de vida y otros. Sería imposible indicar con certeza cuál de ellos fue determinante en el desarrollo de la enfermedad.

Básicamente, lo que se percibe es que el Poder Judicial viene entendiendo que muchas de las decisiones de la vida moderna tienen riesgos inherentes en diversos grados, más que la libertad de escoger de los consumidores sobre cuáles productos desean consumir, lo cual constituye un derecho esencial y principio fundamental para la consolidación del merca-
} do de consumo. ratificar el Convenio, o que por lo menos estuviese dentro de los primeros 40 países en formar un número mínimo para que pudiese entrar en vigor la Convención, Brasil fue uno de los 100 países en ser considerado, casi al final del plazo estipulado, para estar en esta primera sesión con voz y voto. ${ }^{32-34}$

Esta situación contribuyó a que la negociación del Convenio en el Congreso Nacional de Brasil fuera extremadamente más agitada, debido a una intensa y agresiva campaña desinformativa, organizada por la BAT/ Souza Cruz y sus asociados. Con este fin, la BAT fomentó una intensa y agresiva campaña de desinformación sobre ficticios impactos económicos negativos, supuestamente asociados a la ratificación del tratado por parte de Brasil, a fin de generar pánico y rechazo en las 190 mil familias fumicultoras, principalmente en la región sur del país, en donde se concentra más de $90 \%$ de la producción nacional del tabaco. A través de una intensa actividad en los gabinetes parlamentarios brasileños se buscó generar un clima de incertidumbre y de confusión en su arbitraje. Como consecuencia, llevó a varios congresistas a incorporar y mantener el argumento contrario a la ratificación del Convenio Marco, mismo que fue ampliamente refutado por sectores gubernamentales y representantes de la sociedad civil organizada, defensores de la ratificación. ${ }^{35,36}$

Asimismo, esta situación llevó a un liderazgo parlamentario de las comisiones del Senado responsables de analizar la materia, al realizar varias audiencias públicas en regiones fumicultoras. La iniciativa permitió desmentir las distorsiones maliciosamente planteadas sobre la Convención, antes de la garantía final dada por el Congreso Nacional brasileño para la ratificación del tratado. ${ }^{37}$ En este proceso de distorsión de los objetivos del Convenio Marco merece destacarse el papel de la Asociación de Fumicultores del Brasil (AFUBRA), subsidiaria de la International Tobacco Growers Association (ITGA), una organización auspiciada por la BAT según amplia información interna de compañías de humo accesible al público conforme a lo mencionado anteriormente. ${ }^{35,38}$

Sin embargo, este lento proceso consolidó tanto la posición y compromiso de la sociedad brasileña con la Convención y sus objetivos como el entendimiento de todo el pueblo brasileño, incluidos los segmentos sociales que hoy se dedican a la producción agrícola del tabaco, y ratificó que la Convención no debe ser temida, pues busca rescatar a las generaciones presentes y futuras de los graves daños sociales, ambientales y económicos derivadas del consumo del tabaco. ${ }^{39}$

En resumen, todo este proceso puede ser entendido como una tentativa de la BAT de utilizar a Brasil para descalificar el Convenio Marco y, con ello, minar los esfuerzos internacionales que obstaculizan sus pro- 
pios objetivos. Esta tentativa fue frustrada gracias al importante papel que la sociedad civil organizada desempeñó, con su apoyo al poder ejecutivo brasileño en sus gestiones para impulsar la ratificación del Convenio Marco en el Congreso Nacional.

Cabe mencionar que muchas organizaciones nacionales e internacionales apoyaron este proceso actuando más frontalmente, haciendo divulgaciones o movilizando peticiones de apoyo regional. Una mención especial debe ser hecha al papel del Framework Convention Alliance, ${ }^{40}$ sobre todo a sus representaciones, así como a la Interamerican Heart Foundation, la Path Canada, la Rede Tabaco Zero ${ }^{41}$ y la Network for Accountablilty of Tobacco Transnationals (NATT) ${ }_{1}^{42}$ entre otras integrantes. También cabe mencionar a otras organizaciones no gubernamentales del ámbito nacional como la Asociación Médica Brasileña y, en especial, la Asociación Médica de Rio Grande del Sur. La lista de menciones incluye, también, las siguientes organizaciones nacionales, no menos importantes: la Federación de los Trabajadores de la Fumicultura Familiar del Sur (FetrafSul), la Organización Tierra de los Derechos, la Sociedad Paulista de Oncología Clínica, la Sociedad Brasileña de Neumología, la Asociación Brasileña contra el Cáncer, Redes Unidas y la Asociación Brasilera de Salud Colectiva. Este amplio involucramiento de la sociedad civil en la defensa del Convenio Marco refuerza sobremanera la importancia del artículo $4.7,{ }^{43}$ que entre otras cosas dice:

Para alcanzar los objetivos del Convenio y de sus protocolos y aplicar sus disposiciones, las Partes se guiarán, entre otros, por los principios siguientes: [...]

7. La participación de la Sociedad Civil es esencial para conseguir el objetivo del Convenio y de sus protocolos.

El caso del índice de sustentabilidad de la BOVESPA

Siguiendo las tendencias mundiales de valorización de empresas socialmente responsables, la Bolsa de Valores de Brasil (BOVESPA), junto con varias otras instituciones, creó un índice de acciones referenciales para las inversiones socialmente responsables, el ISE (Índice de Sustentabilidad Empresarial). ${ }^{44} \mathrm{Su}$ objetivo es "propiciar un ambiente de inversión compatible con una demanda de desarrollo sustentable de la sociedad contemporánea y estimular la responsabilidad corporativa considerando aspectos de gobernanza corporativa, eficiencia económica, equilibrio ambiental y justicia social". Esta iniciativa está respaldada por la International Finance Corporation y entre otros parámetros sigue los de los índices Dow Jones de sustentabilidad. ${ }^{45}$

En Brasil, diversas organizaciones asociadas a la BOVESPA formaron un Consejo Deliberante que, a su vez, contrató una institución con expertise en esas cues- tiones -el Centro de Estudios de Sustentabilidad de la Fundación Getúlio Vargas (CES-FGV)- para desarrollar un cuestionario que interpretara el funcionamiento de las compañías integrantes de las 150 acciones de la BOVESPA. ${ }^{46}$ Mientras tanto, la Comisión inicialmente decidió no excluir de este proceso selectivo a la industria tabacalera, así como a las industrias bélicas y de bebidas alcohólicas, al contrario de lo que ya venía sucediendo en el plano internacional, como. por ejemplo, en la Bolsa de Londres, el Financial Times, y el Indice Dow Jones de sustentabilidad. Esta decisión provocó una crisis interna en la Comisión y culminó con la salida del Instituto Brasileño de Análisis Sociales y Económicos (IBASE) después de un amplio debate nacional. ${ }^{47}$

Una gran movilización de las organizaciones no gubernamentales, en conjunto con la Secretaría de Salud, a través del Instituto Nacional del Cáncer, culminó con la realización de una audiencia pública en la Asamblea Legislativa de Sao Paulo que llevó a la posterior exclusión de la industria tabacalera del ISE. ${ }^{48,49}$

\section{Conclusiones}

El tema de la responsabilidad social corporativa ha sido un asunto de gran importancia y preocupación en el mundo actual y ha recibido diferentes denominaciones, tales como responsabilidad social, ciudadanía corporativa, desarrollo sostenible, ética corporativa. ${ }^{50}$

En este contexto el concepto de desarrollo sostenible se ha tornado ya muy popular, especialmente a partir del informe ${ }^{51}$ de la Comisión Brundtland de 1987:

Desarrollo que permite a las presentes generaciones entender sus necesidades sin comprometer el entendimiento de las necesidades de futuras generaciones.

Asimismo, ha sido utilizado ampliamente por muchas personas, empresas e instituciones bien intencionadas, y ha representado un paradigma en la orientación de las posturas ecológicamente benéficas en relación a los recursos naturales. Además, según Fernando Fernán$\mathrm{dez},{ }^{52}$ la expresión viene siendo usada actualmente, por lo menos, en cuatro sentidos diferentes:

1. Un elogiable y necesario objetivo (para los de buena fe).

2. Una manera de obtener permiso para explorar recursos en áreas naturales protegidas; todas las puertas oficiales se abren mediante la mágica palabra "sustentabilidad"..

3. Una manera de incluir un número de productos en el mercado cada vez más consciente ecológicamente: muchos productos venden más cuando tienen 
una sola certificación-exploración sustentable aun cuando en realidad no sea o no se sepa qué es.

4. Una forma de desviar otros usos de los abundantes recursos financieros internacionales destinados a a la conservación de la naturaleza.

A estos cuatro sentidos se puede agregar un quinto con el que ilustrar el caso de las empresas tabacaleras que han usado los términos de responsabilidad social y desarrollo sustentable como forma de crear una imagen positiva, desviar la atención de los daños sociales generados por sus productos, captar la atención de asociaciones diversas, sobre todo el apoyo de gobiernos, y así, gracias a todo ello, capitalizar influencias para garantizar la continuidad de sus negocios.

Todo lo expuesto anteriormente exige plantearse una serie de interrogantes: ¿cuál debería ser la posición de las sociedades y los gobiernos ante el hecho de que la producción del tabaco tiene como único y exclusivo fin el consumo humano y de que a ciencia cierta ha demostrado, de forma exhaustiva e inequívoca, el efecto de generar dependencia, enfermedades graves, incapacitadoras y fatales?; ¿qué posición deberían tomar los gobiernos y sociedades contra empresas cuyos productos no traen ningún beneficio al que los consume, sino que, por el contrario, matan al menos a la mitad de sus consumidores?; ¿cuál debería ser la postura de los gobiernos y toda la sociedad contra las actitudes poco claras de tales empresas, en donde al tiempo que buscan presentarse como socialmente responsables y defensoras del desarrollo sostenible actúan de forma frontal o disfrazada para minar iniciativas que reduzcan el consumo de sus productos, iniciativas reconocidas ampliamente hoy como importantes para el desarrollo sostenible y el combate a la pobreza?

Hoy más que nunca se hace necesario que los órganos gubernamentales, así como las diversas representaciones de la sociedad civil organizada, actúen en defensa del desarrollo sustentable. Esto implica entender que junto con la preservación del medio ambiente la salud y la educación representan la base de la productividad económica y que, por tanto, una población saludable es esencial para la reducción de la pobreza, el crecimiento económico y el desarrollo sustentable. Afortunadamente, asistimos hoy en día a un movimiento formidable para promover el consumo responsable. En palabras del Instituto Kairos: ${ }^{53}$ "La capacidad de cada persona o institución pública o privada de escoger y/o producir servicios y productos que contribuyan, en forma ética y de hecho, para mejorar la calidad de vida de cada una de las sociedades y de su ambiente". Se trata de un movimiento social que demuestra todo el poder de la sociedad civil para promover transformaciones positivas guiadas por este concepto del desarrollo sustentable.
Una acción solamente surte efecto cuando todas esas instancias participan de forma orquestada y comprometida con el futuro de nuestro planeta y las generaciones futuras, y promueve la necesidad de un trabajo en red que integre las diversas esferas de la sociedad. Las diferentes experiencias positivas de Brasil en la lucha contra las estrategias de la industria tabacalera demuestra el éxito de esta amplia articulación en red, la cual ha ejercido un papel fundamental en el monitoreo y fiscalización de las políticas públicas para el control del tabaco. En este sentido, el Convenio Marco representa la esencia de un amplio trabajo en red a escala internacional, que nace de la convicción de que el control mundial del consumo de tabaco depende de la unión de esfuerzos trasnacionales, tanto gubernamentales como no gubernamentales, para "proteger a las generaciones presentes y futuras de las devastadoras consecuencias sanitarias, sociales, ambientales y económicas generadas por el consumo y la exposición del tabaco".

Es importante resaltar que el artículo 5.3 del Convenio Marco representa el compromiso de protegerlo de la interferencia indebida de la industria tabacalera. Esto conlleva a un gran desafío, sobre todo para los países que ratificaron el Convenio Marco: cómo manejar las interfaces de relación que tienen que mantener con la industria tabacalera, sin permitir interferencias indebidas en su política sobre el control del tabaquismo.

Esa fue una de las preocupaciones manifestadas por muchas de las delegaciones durante la Primer Conferencia de Partes del Convenio Marco (COP1), celebrada en febrero del 2006, y que llevó al acuerdo sobre la necesidad de trazar directrices que orientaran a los gobiernos con mejores prácticas para el cumplimiento del mencionado artículo 5.3..$^{54}$

\section{Referencias}

I. World Health Organization. Tobacco or Health Program Guidelines for controlling and monitoring the tobacco epidemic. Ginebra, Suiza:WHO, 1996. 2. Doll R, Peto R, Wheatley $\mathrm{K}$ et al. Mortality in relation to smoking: 40 years'observations on male. British doctors. BMJ I994;309: 90 I-9I0.

3. U.S. Surgeon General. Reducing the Health consequences of smoking. 25 years of progress. A report of the Surgeon General Rockville, Maryland: U.S. Department of Health and Human Services. Public Health Service, Centers for Disease Control, Centers for Chronic Disease Prevention and Health Promotion. Office on Smoking and Health, 1989. 4. Rosemberg J. 2002. Pandemia do Tabagismo - Enfoques históricos e atuais. São Paulo: Secretaria de Saúde de São Paulo-Centro de Vigilância Epidemiológica, 2002.

5. Guindon GE, Boisclair D. Past, Current and Future Trends in Tobacco Use. In: Health, Nutrition, and Population Family (HNP) of the World Bank's Human Development Network (HNP) Discussion Paper. Economics of Tobacco Control Paper no. 6. Washington DC:WB, 2003. Disponible en: http://www.terra.com.br/istoe//776/I776vermelhas.htm 6. OECD (Organization for Economic Cooperation and Development \& WHO (World Health Organization). Poverty and Health (draft). Reference document published by OECD and WHO). 2001.

7. World Health Organization. Tobacco and poverty: a vicious circle". Ginebra, Suiza:WHO, 2004.Disponible en: http://www.who.int/tobacco/ communications/events/wntd/2004/en/ [Consultado el 2006 abril 5.] 
8.WHA 54.18 - FIFTY -FOURTHWORLD HEALTH ASSEMBLY 2001 Agenda item 13.5. Transparency in tobacco control process 22 May 200I. 200I. Disponible en: http://www.who.int/tobacco/framework/ wha_eb/wha54_I8/en/index.html. Consultado el I de febrero de 2006. 9. World Bank. Economics of Tobacco Control. What is the World Bank's Policy on Tobacco? Washington DC:WB, 200I. Disponible en: http:// wwwl.worldbank.org/tobacco/aboutreport.asp [Consultado en octubre de 2005].

10. World Bank. Development in practice. Curbing the Epidemic. Governments and the Economics of Tobacco Control. Washington, DC: WB, 1999.

II.World Health Organization. Tobacco and poverty: a vicious circle". Ginebra, Suiza:WHO, 2004. Disponible en: http://www.who.int/tobacco/ communications/events/wntd/2004/en/ [Consultado el 5 de abril de 2006]. 12. World Health Organization. Disponible en: http://www.who.int/ tobacco/media/en/unreportl.pdf

13. World Health Organization. Disponible en: http://www.who.int/ tobacco/global_interaction/un_taskforce/meeting_2005/en/index.htm 14. UNCTAD XI. Globalization of the Tobacco Epidemia-Tobacco Control and Development. Disponible en: http://www.unctadxi.org/templates/ Event_525.aspx. y en http://www.who.int/tobacco/communications/ events/unctad2004/en/. [Consultado el 05 de abril de 2006].

I5. ECOSOC 2004 Resolution - Tobacco Control. Disponible en: http:// www.who.int/tobacco/communications/events/2004/ecosoc_resolution/ en/. [Consultado el 06 de abril de 2006]

16. Disponible en: http://legacy.library.ucsf.edu/

17. World Health Organization. The tobacco industry documents. What they are, what they tell us, and how to search them. A practical manual. Washington DC:WHO, 2002. Disponible en: www.who.int/tobacco/ communications/TI_manual_content.pdf [Consultado el 04 abril de 2006]. 18. Souza Cruz. Responsabilidade social. Disponible en: http://www.souzacruz.com.br/OneWeb/sites/SOU_5RRP92.nsf/ vwPagesWebLive

80256DAD006376DD80256D87005B $19 \mathrm{BB}$ ?opendocument\&SID=\&DTC=. [Consultado el 03 abril de 2006].

19. Campaign for Tobacco Free Kids \& Action on Smoking and Health

(ASH). Confíe en nosotros. Somos la industria tabacalera. Washington, DC:ASH, $200 \mathrm{I}$

20. Action on Smoking and Health \& Christian Aid. BAT in its own words. ASH, 2005. Disponible en: http://www.ash.org.uk/html/conduct/ pdfs/bat2005.pdf. [Consultado en Febrero 2006]

2I. Departamento de Estudos Sócio-Econômicos Rurais. A Cadeia Produtiva do Fumo. Contexto Rural,Ano III, no 4, dezembro. DESER, 2003.

22. Cavalcante T. Experiencia brasileña con políticas de control del tabaquismo. Salud Pub Méx 2004;46; 549-558.

23. ISTO É. Entrevista on-line. Flávio de Andrade. Missão Quase Impossível. O presidente da Souza cruz, fumante moderado enfrenta ao mesmo tempo o avanço de antitabagistas e a prosperidade dos ilegais. I5/I0/ 2003

24. Souza Cruz Processos Judiciais. Disponible en:

http://www.souzacruz.com.br/OneWeb/sites/SOU_5RRP92.nsf/ vwPagesWebLive/

80256DAD006376DD80256DA500408227? opendocument\&SID=\&DTC=.

[Consultado el 08 de abril de 2006].

25. Souza Cruz. Pode uma empresa de cigarros ser socialmente responsável? Disponible en: http://www.souzacruz.com.br/OneWeb/ sites/SOU_5RRP92.nsf/rwPagesWebLive/

80256DAD006376DD80256D87006A835E!opendocument\&SID=\&DTC= 26. Instituto Nacional de Câncer (INCA). Disponible en: http://www.inca.gov.br/tabagismo

frameset.asp?item=economia\&link=novaregulamentacao.htm

27. Banco Mundial.A epidemia do tabagismo. Os governos e os aspectos econômicos do controle do tabaco. Washington, DC: BM, 2000

28. Disponible en: http://www.cni.org.br/adins/33II.htm http:// www.idec.org.br/noticia.asp?id=2947

29. Fundo Justiça Sem Papel. Disponible en:

http://www.justicasempapel.org.br/

index.php?option=com_content\&task=view\&id= $|8 \&|$ temid $=47$.

[Consultado el 06 de abril de 2006].

30. Souza Cruz. Processos Judiciais. Disponible en: http://www. souzacruz.com.br/OneWeb/sites/SOU_5RRP92.nsf/vwPagesWebLive/ 80256DAD006376DD80256DA50040C9EC? opendocument\&SID=\&DTC=
31. Consultor jurídico. Parceria cuestionada. MPF recorre de decisão que liberou o Justiça Sem Papel. Revista Revista Consultor Jurídico, I3 de fevereiro de 2006 Disponible en: http://conjur.estadao.com.br/static/ text/4|89I,I. [Consultado el 08 de abril de 2006].

32. Disponible en: http://www.cruzazul.org.br/legislacao/laws.asp?id=2 http://gazeta.via.com.br/

default.php?arquivo=_noticia.php\&intldEdicao $=295$ \&intldConteudo $=16679$ http://gazeta.via.com.br/

default.php?arquivo=_noticia.php\&intldEdicao $=296 \&$ \&intldConteudo $=16752$

33. Disponible en: http://www.terradedireitos.org.br/ index.php?pg=conteudo\&tema $=4 \&$ conteudo_id $=219 \&$ tipo $=1$

34. Disponible en: http://www.senado.gov.br/agencia/ verNoticia.aspx? $\operatorname{codNoticia}=5|38| \& \operatorname{cod}$ Aplicativo $=2$ 35. Ministério da Saúde. Secretaria de Atenção à Saúde. Instituto Nacional de Câncer. A ratificação da Convenção-Marco para o Controle do Tabaco pelo Brasil: mitos e verdades. Rio de Janeiro: INCA, 2004. Disponible en: http://www.inca.gov.br/tabagismo/cMarco3/ mitos_verdades.pdf. Accesado el 03 abr 2006.

36. Johns P.Audiência pública para debater o tratado internacional de controle do tabaco vira palanque político. Ponto de Vista. Revista do Terceiro Setor , 02 de setembro de 2005. Disponible en: http://arruda.rits.org.br/notitial/servlet newstorm.notitia.apresentacao.ServletDeSecao? codigoDaSecao= | |\&dataDojornal=| | 25694829000. Accesado el 09 de abril de 2006. 37. Disponible en: http://obid.senad.gov.br/OBID/Portal/ conteudo.jsp?ldPJ $=257 \mid$ \&ldEC $=4203$

38. ASH ( ACTION ON SMOKING AND HEALHT) \& CHRISTIAN AID (2005) . BAT in its own words. Disponible en: http://www.ash.org.uk/html/conduct/pdfs/bat2005.pdf. Febrero 2006 39. Gewer A. Federação dos Trabalhadores da Agricultura Familiar do Sul. Leia sobre a Convenção Marco : Derrotamos o lobby e o proselitismo político". 2005. Disponible en: http://www.fetrafsul.org.br/ index.php?option $=$ com content\&task $=$ view\&id $=|9|$ \& $\mid$ temid $=0$

Accesado el 09 de abril de 2006

40. Disponible en: http://www.fctc.org/

4l. Disponible en: http://www.tabacozero.net/a_plataforma.asp

42. Disponible en: http://www.treatycheck.org/NATT.htm

43. World Health Organization. Framework Convention on Tobacco Control. Ginebra, Suiza:WHO, 2003. Disponível em: http://www.who.int/ tobacco/framework/download/en/.Acessado em: 03 abr 2006. 44. BOVESPA. Disponible en: http://www.bovespa.com.br/Noticias/ 05072INotA.asp

45. Centro de estudos em sustentabilidade da eaesp/Fundação Getúlio Vargas. Índice de sustentabilidade empresarial BOVESPA 2005. Documento preparatório para a Audiência Pública de 10 de agosto de 2005

46. BOVESPA. Disponible en: http://www.bovespa.com.br/Mercado/ RendaVariavel/Indices/FormConsultaApresentacaoP.asp?!ndice=ISE 47. Disponible en:http://www.fiomaravilha.com.br/assinaturas/29.1 I.05-3.htm 48. O GLOBO - RAZÃO SOCIAL. O Espaço da Empresa cidadã.

Entrevista Constantino Mendonça, diretor da Souza Cruz. Março 2006 no 34.

49. IBASE. Olhar sobre índice da Bovespa. Publicado en 09//2/2005.

Disponível em http://www.ibase.br/pubibase/cgi/cgilua.exe/sys/ start.htm? sid=127\&infoid=| 190 Acessado em 09 de abril de 2006 50. ISO. Corporate social responsability. Concepts and solutions. ISO committee on consumer policy. Porto f Spain (Trindad), 10 June 2002 5I. Brundtland GH.. Our Common Future. World Commission on Environment and Development. Oxford, 1987.

52. Fernandez F.Aprendendo a lição de Chaco Canyon: do "Desenvolvimento Sustentável" a uma Vida Sustentável. Instuto Ethos Reflexão agosto de 2005. Disponible en: http://www.uniethos.org.br/ DesktopDefault.aspx?TabID=3708\&Alias=uniethos\&Lang=pt-BR .

Accesado el 08 de abril de 2006

53. Istituto Kairós. O que é consumo responsável? Disponible en:http://www.institutokairos.org/Consumo\%20Responsável/ consumo_responsavel.principal.htm . Accesado el 09 de abril de 2006 54. World Health Organization. Disponible en: http://www.who.int/gb/ fctcl - A/FCTC/COP/I/II 